\title{
Periodontal Disease in Diabetes Mellitus: A Case- Control Study in Smokers and Non-Smokers
}

\author{
Emese Battancs · Dorottya Gheorghita $\cdot$ Szabolcs Nyiraty \\ Csaba Lengyel · Gabriella Eördegh · Zoltán Baráth • Tamás Várkonyi • \\ Márk Antal (D)
}

Received: July 11, 2020 / Accepted: September 15, 2020 / Published online: September 25, 2020

(C) The Author(s) 2020

\begin{abstract}
Introduction: It is well established that periodontal disease (PD) and diabetes mellitus (DM) can have a detrimental effect on each other's disease course, and that cigarette smoking exacerbates both conditions. However, literature on the periodontal status of smokers with DM is scarce, and the studies conducted to date did not use healthy controls or non-smokers with DM as controls. Consequently, the individual effects of smoking and DM on PD are difficult to untangle and estimate.
\end{abstract}

Methods: A total of 128 participants were recruited to this study and their data analyzed. They were assigned to four groups: smoking

E. Battancs · D. Gheorghita $\cdot$ M. Antal $(\bowtie)$ Department of Esthetic and Operative Dentistry, Faculty of Dentistry, University of Szeged, Szeged, Hungary

e-mail: antal.mark@szte.hu

S. Nyiraty · C. Lengyel · T. Várkonyi

Department of Internal Medicine, Faculty of

Medicine, University of Szeged, Szeged, Hungary

G. Eördegh

Faculty of Health Sciences and Social Studies,

University of Szeged, Szeged, Hungary

Z. Baráth

Department of Prosthodontics, Faculty of Dentistry,

University of Szeged, Szeged, Hungary patients with DM (SDM); non-smoking patients with DM (NSDM); smokers without DM (control group, SC) and (4) non-smokers without DM (control group, NSC). Each group consisted of 32 age-matched participants. The periodontal status of the participants was assessed by full oral examination. To express periodontal status, we used the four-stage classification introduced by Fernandes and colleagues (J Periodontol. 80(7):1062-1068, 2009). The control of DM was estimated by measuring hemoglobin A1c (HbA1c) levels in the peripheral blood.

Results: A significant difference in the severity of PD was found between the SC and NSC groups $(p=0.027)$ and between the NSC and SDM groups $(p=0.000)$, while the difference between the NSDM and SDM groups approached significance $(p=0.052)$. No person in the smoker groups could be classified as having a healthy periodontal status. The four-stage classification followed a normal distribution in the healthy, non-smoking controls (NSC). Smoking caused a shift toward medium-severe PD, while a marked shift toward the most severe stage was observed when both smoking and DM were present (SDM). There was no significant association between the type of DM and periodontal status, nor between diabetes control and the severity of PD. Persons in the SDM group had significantly fewer teeth than those in the NSC group (mean \pm standard deviation: $16.0 \pm 7.9$ vs. $20.7 \pm 5.6 ; p=0.02)$. 
Conclusion: Smoking damages the periodontium of even healthy individuals, but the damage is multiplied in a smoker who has DM, even though the effect of DM alone on periodontium health is relatively mild. Our results suggest a synergy between DM and smoking in terms of damage to the periodontal tissues, but the limited sample size of this study does not allow any hard conclusion to be drawn.

Keywords: Comorbidity; Inflammation; Periodontal diseases; Smoking; Type 1 diabetes mellitus; Type 2 diabetes mellitus

\section{Key Summary Points}

Why carry out this study?

Periodontal disease (PD) and diabetes mellitus (DM) are known to detrimentally affect each other's disease course, and cigarette smoking independently exacerbates both conditions.

Existing literature on the periodontal status of patients with DM who smoke is scarce, and studies conducted to date did not use healthy controls or non-smoking patients with DM as controls; consequently, the individual effects of smoking and DM on PD are difficult to estimate.

We hypothesized that the periodontal status of patients with DM with no smoking history would be significantly poorer than that of healthy non-smoking controls, and that periodontal status in the former would be further exacerbated by smoking.

\section{What was learned from the study?}

The periodontal status of 128 participants categorized into four groups, namely smoking patients with DM (SDM), nonsmoking patients with DM (NSDM), (3) smokers without DM (control group, SC) and non-smokers without DM (control group, NSC), was assessed.
A significant difference in severity of $\mathrm{PD}$ was found between the SC and NSC $(p=0.027)$ groups and between the NSC and SDM $(p=0.000)$ groups, while the difference between the NSDM and SDM groups was nearly significant $(p=0.052)$. In the smoker groups, no one could be classified as having a healthy periodontal status.

In this study, smoking caused a shift toward medium-severe PD, while a marked shift toward the most severe stage was observed when smoking was combined with DM.

\section{DIGITAL FEATURES}

To view digital features for this article go to https://doi.org/10.6084/m9.figshare.12957311.

\section{INTRODUCTION}

Studies have established that periodontal status and systemic diseases have an effect on each other [1]. The most studied interactions are those between periodontal disease (PD) and cardiovascular conditions [2], rheumatoid arthritis [3-5] and diabetes [6-8], but relationships between PD and hypertension [8], osteoporosis [9], psoriasis [10, 11] pulmonary diseases [12], pregnancy and perinatal complications [13-15] have also been shown.

$\mathrm{PD}$ is a chronic, destructive oral condition characterized by infection-related inflammation of the tooth-supporting tissues, leading to bone destruction and tooth loss [16]. Excessive inflammation of the periodontal tissues (periodontitis) is the hallmark feature of PD. In PD, bacteria in the oral biofilm interfere with the body's defense mechanisms, activating the immune system which in turn results in inflammation and the immune response $[17,18]$. The levels of C-reactive protein, prostaglandin $\mathrm{E}_{2}$ interleukin-1beta, and tumor 
necrosis factor alpha increase in PD [19]. A similar increase in the presence and concentration of these inflammatory mediators has also been observed in persons with diabetes mellitus (DM) $[20,21]$.

In type 1 diabetes (T1D), the pancreas is unable to produce (enough) insulin, whereas type 2 diabetes (T2D) is characterized by insulin resistance often combined with a relatively low level of insulin secretion. According to the World Health Organization, the prevalence of DM worldwide in 2014 was $8.5 \%$ [22]. DM has several known complications, including retinopathy, nephropathy, neuropathy, macrovascular abnormalities, prolonged wound healing and PD [23]. The increasing number of recent studies that have focused on examining the relationship between DM and PD testified to a definite surge in scientific interest [24-26]. Given that certain cytokines and other inflammatory mediators are involved in both diseases, DM and PD appear to have a mutually detrimental effect on each other. A strong correlation was found between PD and DM [27-29], as well as between PD and the metabolic control of DM both in T1D and T2D [30-36]. According to some authors, this relationship is two-way [37-39], which has led to the notion that periodontal therapy may influence DM outcomes $[40,41]$. In support of this, a positive effect of periodontal therapy on DM outcomes has been observed in various studies [41-44].

Smoking is a harmful and addictive habit which, in addition to being an addiction, gives rise to or exacerbates various pathological conditions [45-48], including DM and PD. Obradovic and co-workers studied smokers and nonsmokers with T1D or T2D who also had PD and found that smoking had an adverse effect on the periodontal status of these patients. An obvious weakness of that study was that only patients with PD were enrolled in the study and, consequently, no comparison was made with healthy controls [49]. Gupta and colleagues investigated the relationship between T2D and smoking in patients with PD and observed that the periodontal status of these patients with PD was significantly worse in the group of smokers with DM than in the group of non-smokers with T2D [50]. Obrak and co-workers found that the periodontal status of non-smokers with T2D with and PD was better than that of their smoking counterparts [51], while Javed and coworkers found that both DM and smoking had a detrimental effect on the periodontal status of patients [52]. These studies draw a hypothetical picture in which DM damages the integrity of the periodontium and smoking likely boosts this effect. Unfortunately, none of these aforementioned studies used healthy controls, so their results cannot be regarded as strong evidence supporting the hypothesis they set up to test. It should also be noted that the relationship between smoking and periodontal status has not yet been investigated and compared to healthy controls in patients with T1D or T2D who have good periodontal health. The aim of this study was to fill the gaps in the literature by examining the relationship between smoking and periodontal status in both patients with DM (T1D or T2D) and healthy controls.

Our research group has been investigating the relationship between various systemic diseases for a decade, including systemic conditions such as psoriasis and rheumatoid arthritis, and we are especially interested in the additional effect of smoking [25]. In 2014, we first proposed that smoking may act as a permissive factor of PD in patients with psoriasis [11], and we subsequently demonstrated something similar in rheumatoid arthritis [53]. In the exploratory study reported here, we hypothesized that (1) the periodontal status of patients with DM with no smoking history would be significantly poorer than that of healthy nonsmoking controls, and (2) this effect would be exacerbated by smoking. The specific question we sought an answer to was whether PD occurs more often and in a more serious form in smokers with DM than in non-smokers with $\mathrm{DM}$. Thus, the aim of the this study was to find evidence for or against the permissive/boosting effect of smoking on periodontal destruction in patients with DM. The effect of various subfactors (such as the type of DM and the success of blood glucose control) was also considered. 
Table 1 Descriptive statistics of the patient and control groups

\begin{tabular}{|c|c|c|}
\hline Descriptive statistic of the patient and control groups & Diabetes patient groups & Control groups \\
\hline \multicolumn{3}{|l|}{ Age (years) } \\
\hline Smoker subsample & $54.5 \pm 13.4$ & $54.0 \pm 13.6$ \\
\hline Non-smoker subsample & $54.3 \pm 13.9$ & $54.5 \pm 13.9$ \\
\hline \multicolumn{3}{|l|}{ Sex ratio } \\
\hline Non-smoker subsample & 18F:14 M (56\%:44\%) & 20F:12 M (62\%:38\%) \\
\hline Smoker subsample & 18F:14 M (56\%:44\%) & 22F:10 M (69\%:31\%) \\
\hline \multicolumn{3}{|l|}{$\mathrm{CAL}(\mathrm{mm})$} \\
\hline Smoker subsample & $2.94 \pm 1.60$ & $2.51 \pm 1.32$ \\
\hline Non-smoker subsample & $2.83 \pm 1.51$ & $2.50 \pm 1.24$ \\
\hline \multicolumn{3}{|l|}{$\mathrm{PPD}(\mathrm{mm})$} \\
\hline Smoker subsample & $2.60 \pm 1.25$ & $2.27 \pm 1.06$ \\
\hline Non-smoker subsample & $2.71 \pm 1.28$ & $2.30 \pm 0.98$ \\
\hline \multicolumn{3}{|l|}{ Number of teeth } \\
\hline Smoker subsample & $16 \pm 8.0$ & $19.2 \pm 7.5$ \\
\hline Non-smoker subsample & $18 \pm 7.9$ & $20.7 \pm 5.7$ \\
\hline T1D subsample & $22 \pm 6.5$ & NA \\
\hline T2D subsample & $16.2 \pm 7.7$ & NA \\
\hline
\end{tabular}

Values in table are presented as the mean \pm standard deviation (SD) or as frequencies (number of observations with the percentage in parenthesis)

$C A L$ Clinical attachment level (distance between the cemento-enamel junction and the bottom of the pocket), $F$ female, $M$ male, PPD probing pocket depth (distance between the marginal gingiva and the bottom of the periodontal pocket), T1D/ $T 2 D$ type 1 type 2 diabetes

\section{METHODS}

\section{Patients}

Patients with DM were recruited form the Department of Internal Medicine of the University of Szeged, Faculty of Medicine, Szeged, Hungary, and healthy controls were recruited from the Csongrád County Pulmonary Screening Station, Szeged, Hungary in 2018-2019. Before enrollment, all participants had been informed about the study. Participation was voluntary, and written informed consent was obtained from each participant. The protocol of the study conformed in all respects to the tenets of the Declaration of Helsinki of
1964, and its later amendments, and the study was approved by the Regional Research Ethics Committee for Medical Research at the University of Szeged, Hungary (144/2014-B/001, accepted 2014, modified in 2019).

A total of 128 participants were enrolled in the study. These participants were assigned to four groups: (1) smoking patients with DM (SDM); (2) non-smoking patients with DM (NSDM); (3) smokers without DM (control group, SC) and (4) non-smokers without DM (control group, NSC). Each group consisted of 32, age-matched participants. Gender matching was also possible in most cases, but females were over-represented in both the patient and the control groups. Detailed descriptions of the 
Table 2 Disease duration, complications and co-morbidities among patients with diabetes

\begin{tabular}{|c|c|c|}
\hline $\begin{array}{l}\text { Disease duration, } \\
\text { complications and co- } \\
\text { morbidities }\end{array}$ & $\begin{array}{l}\text { Smokers } \\
\text { with } \\
\text { diabetes }\end{array}$ & $\begin{array}{l}\text { Non-smokers } \\
\text { with diabetes }\end{array}$ \\
\hline Duration of disease (years) & $\begin{array}{l}18.56 \\
\quad \pm 7.8\end{array}$ & $\begin{array}{l}18.72 \\
\quad \pm 10.03\end{array}$ \\
\hline T1D & $10(31.3 \%)$ & $12(37.5 \%)$ \\
\hline $\mathrm{T} 2 \mathrm{D}$ & $22(68.8 \%)$ & $20(62.5 \%)$ \\
\hline Diabetic nephropathy & $8(25 \%)$ & $11(34.4 \%)$ \\
\hline Diabetic retinopathy & $3(9.4 \%)$ & $12(37.5 \%)$ \\
\hline Diabetic polyneuropathy & $10(31.3 \%)$ & $15(46.9 \%)$ \\
\hline $\begin{array}{l}\text { Chronic obstructive } \\
\text { pulmonary disease }\end{array}$ & $2(6.3 \%)$ & $3(9.4 \%)$ \\
\hline Asthma & $2(6.3 \%)$ & $2(6.3 \%)$ \\
\hline
\end{tabular}

Values in table are presented as the mean $\pm S D$ or as frequencies (number of observations with the percentage in parenthesis)

group characteristics are given in Tables 1 and 2 . Patients with T1D or T2D were included in the study. Participants in the control group were free of any systemic disease. Patients in the DM groups were compared to age- and gender-matched controls.

The exclusion criteria for all groups were determined based on relevant literature and included obesity (body mass index $\geq 30 \mathrm{~kg} / \mathrm{m}^{2}$ ), excessive alcohol consumption, drug abuse, rheumatoid arthritis and diseases causing neutropenia and local or systemic inflammatory conditions (other than DM) [54]. Poor oral hygiene, defined as a Simplified Oral Health Index (OHI-S) score $>3$ [55] was also an exclusion criterion. Pregnant women, patients receiving cancer therapy, toothless patients or patients with $<3$ teeth, patients with a serum creatinine level $\geq 1.6 \mathrm{mg} / \mathrm{dL}$ and patients with hemoglobinopathy or any other type of hemorrhagic disorder were excluded from the study. Patients in need of antibiotic prophylaxis for any reason or who had received antibiotics within 2 weeks prior to study initiation were also excluded, as were individuals wearing orthodontic braces. Demographic and tobacco use data were collected by means of a questionnaire. A smoker was defined as a person who reported smoking at least two cigarettes for an uninterrupted period of 1 year prior to study initiation. A non-smoker was defined as a person with no self-reported tobacco use for 30 years prior to study initiation. Former smokers were not included in the sample as the category is rather vague (especially when talking about self-reported tobacco use); in addition, the relatively limited sample size would not have allowed a meaningful analysis of an additional category. Smokers in both patient and control groups were comparable in terms of smoking history expressed in pack-years (mean \pm standard deviation [SD] pack-years: patients $17.19 \pm 15.81$, controls $16.47 \pm 13.70$ ). Medical information on both controls and patients with DM was extracted from patient files and hospital records.

The required sample size was calculated with $G^{*}$ Power version 3.1. 5. (University of Kiel, Kiel, Germany), a software program designed especially for statistical power and sample size computation [56]. The software allows the computation of achieved statistical power (posthoc) and required sample size (a priori). As mostly categorical variables were to be analyzed, a priori sample size estimation was performed for crosstabs/chi square/contingency tables, with the following input parameters: effect size $(w): 0.3 ; \alpha$ : 0.05 ; power $(1-\beta)$ : 0.82 ; $d f: 3$. Required sample size was calculated to be $n=128$ (for four groups: DM smoker/DM nonsmoker; control smoker/control non-smoker). It should be noted that the study was powered for the hypothesis tests defined in the section "Statistical Analysis"; more complicated analyses (e.g. regression analyses with multiple predictor variables) were not possible with this sample size. Consequently, this study must be regarded as exploratory in nature.

\section{Parameters Studied}

Control of diabetes was estimated by measuring hemoglobin A1c (HbA1c) levels in the 
Table 3 Severity of periodontal disease by study group

\begin{tabular}{llcrr}
\hline Study groups $^{\mathbf{a}}$ & \multicolumn{4}{l}{ Fernandes et al. classification of PD [16] } \\
\cline { 2 - 5 } & Healthy periodontal status & Early PD & Moderate PD & Severe PD \\
\hline NSC & $4(12 \%)$ & $11(34 \%)$ & $12(37 \%)$ & $5(16 \%)$ \\
SC & $0(0.0 \%)$ & $3(9 \%)$ & $19(59 \%)$ & $10(31 \%)$ \\
NSDM & $2(6 \%)$ & $2(6 \%)$ & $19(59 \%)$ & $9(28 \%)$ \\
SDM & $0(0 \%)$ & $1(3 \%)$ & $11(34 \%)$ & $20(63 \%)$ \\
\hline
\end{tabular}

Values in table are presented as the frequency (number with percentage in parentheses)

$P D$ Periodontal disease

a Study groups categorized by periodontal disease: SDM, smoking patients with DM; NSDM, non-smoking patients with DM; SC, smokers without DM (control group); NSC, non-smokers without DM (control group)

peripheral blood [57]. Diabetes was considered to be well controlled at HbA1c $\leq 7 \%$, moderately controlled at HbA1c $>7.01$ and $\leq 8.5 \%$ and poorly controlled at $\mathrm{HbA} 1 \mathrm{c}>8.5 \%$.

The periodontal status of the patients was assessed by full oral examination, where probing pocket depth (PPD; distance between the marginal gingiva and the bottom of the periodontal pocket, in millimeters), bleeding on probing (BOP; bleeding that is induced by gentle manipulation of the tissue at the depth of the gingival sulcus with a periodontal probe), clinical attachment loss (CAL; distance between the cemento-enamel junction and the bottom of the pocket, in millimeters) and the amount of plaque were evaluated. PPD, BOP, CAL and the amount of plaque was measured at six locations per tooth (mesiobuccally, buccally, distobuccally, distolingually, lingually and mesiolingually), with the exception of the wisdom teeth (which were not evaluated in this study). Williams probes (Hu-Friedy Manufacturing Co. Chicago, IL, USA) were used [58]. Assessment of the clinical severity of PD is still a matter of debate, and several assessment methods are described in the literature for various purposes, from clinical use to epidemiological work [59]. In this study, we used the classification introduced by Fernandes et al. [16]: (1) the participant is considered to have a healthy periodontal status if no CAL or BOP can be detected; (2) early PD is defined as CAL $\geq 1 \mathrm{~mm}$ at $\geq 2$ teeth; (3) moderate PD is defined as CAL $\geq 4 \mathrm{~mm}$ at 3 locations and $\mathrm{PPD} \geq 3 \mathrm{~mm}$ in at least 2 locations; (4) severe PD is defined as CAL $\geq 6 \mathrm{~mm}$ at $\geq 2$ teeth and PPD $\geq 5 \mathrm{~mm}$ at $\geq$ 1 location. To characterize the groups in more detail, we calculated and recorded PPD and CAL separately for each participant, as recommended in other studies [60, 61].

\section{Statistical Analysis}

Statistical analyses were performed in SPSS version 21.0 statistical software (IBM Corp., Armonk, NY, USA). Continuous variables were described as means $\pm \mathrm{SD}$, and categorical variables were characterized as frequencies. For hypothesis testing, one-way analysis of variance (ANOVA; with Tukey's post-hoc test) or Kruskal-Wallis ANOVA (with Mann-Whitney $U$ tests for the pairwise comparisons) were used, depending on whether the normality and homogeneity assumptions were fulfilled. To test the association between categorical variables, we used the chi-square test. If a significant association was found, the dataset was further analyzed with Kruskal-Wallis ANOVA (with Mann-Whitney $U$ tests for the pairwise comparisons). The general limit of significance was set at $p=0.05$, but it was corrected for multiple comparisons with the Bonferroni correction where necessary. 

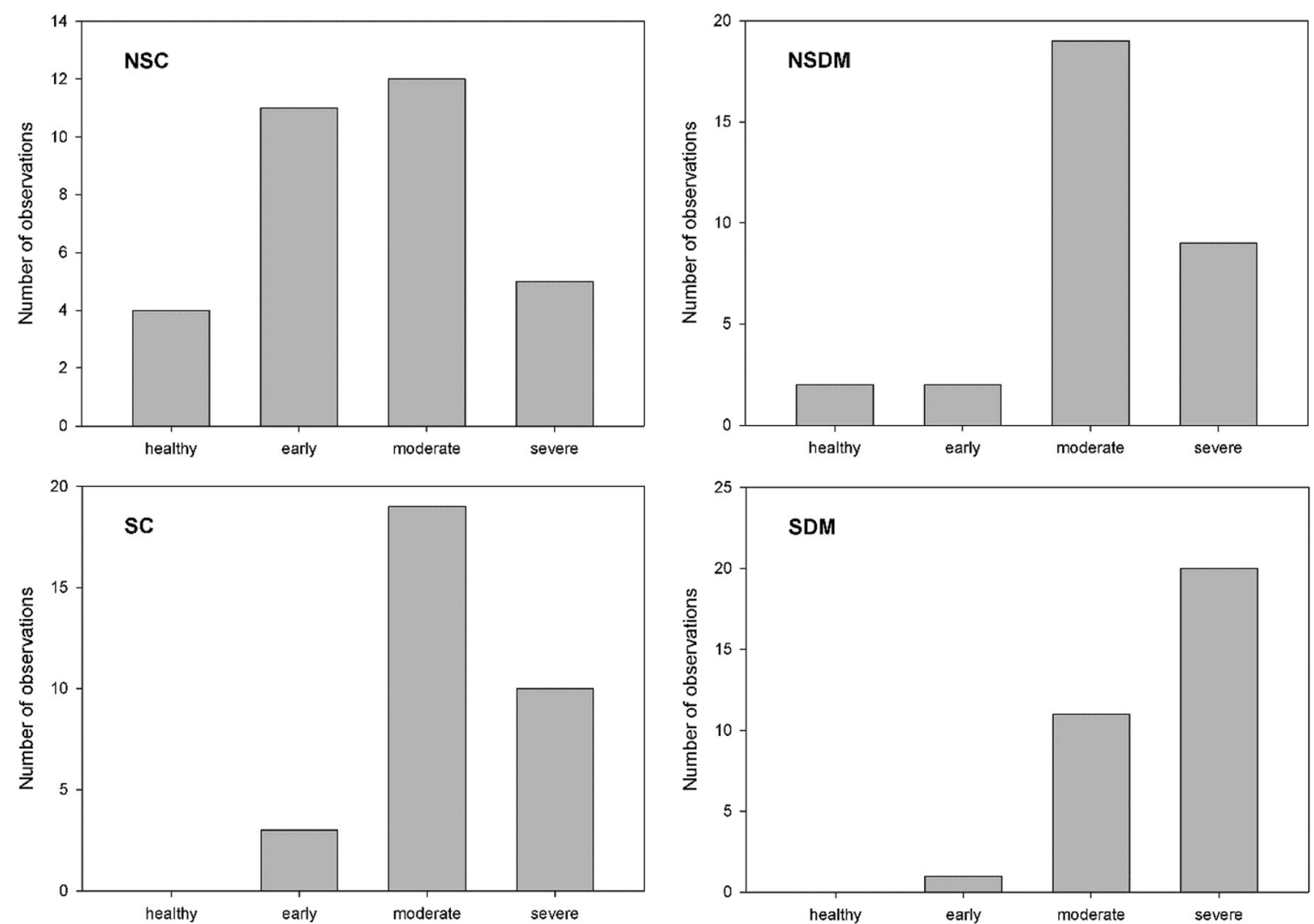

Fig. 1 Distribution of severity stages of periodontal disease across groups (Fernandes et al. classification of PD [16]). Note that while the severity stages follow a normal distribution in healthy, non-smoking controls, smoking causes a definite shift to the right, and when smoking is

combined with diabetes, an extreme shift to the right can be observed. SDM Smoking patients with DM, NSDM non-smoking patients with DM, $S C$ smokers without DM (control group), NSC non-smokers without DM (control group)

Table 4 Severity of periodontal disease by glycemic control as assessed by hemoglobin A1c status

\begin{tabular}{lllll}
\hline HbA1c status $^{\mathbf{a}}$ & Healthy periodontal status & Early PD & Moderate PD & Severe PD \\
\hline WC & $1(6.3 \%)$ & $0(0.0 \%)$ & $9(56.3 \%)$ & $6(37.5 \%)$ \\
MC & $01(4.0 \%)$ & $2(8.0 \%)$ & $12(48.0 \%)$ & $10(40.0 \%)$ \\
PC & $0(0.0 \%)$ & $1(4.3 \%)$ & $9(39.1 \%)$ & $13(56.5 \%)$ \\
\hline
\end{tabular}

Values in table are presented as the frequency (number with percentage in parentheses)

${ }^{a}$ Hemoglobin Alc (HbA1c) status: WC (well-controlled): HbAlc $\leq 7 \%$; MC (moderately controlled): HbA1c $>$ $7.01 \%, \leq 8.5 \%$; PC (poorly controlled): HbAlc $>8.5 \%$

\section{RESULTS}

\section{Periodontal Status by Group}

Group membership was significantly associated with the severity of PD $\left(\chi^{2}=36.910, p=0.000\right.$; see also Table 3). Subsequent analysis of the data using Kruskal-Wallis ANOVA revealed significant variance across the four groups in terms of severity of PD $(H(3)=25.659, p=0.000)$. The pairwise comparisons showed a significant difference in severity of PD between the SC and 
NSC groups $(p=0.027)$ and between the NSC and SDM groups $(p=0.000)$; the difference between the NSDM and SDM groups was nearly significant $(p=0.052)$. Of note is that no one in the two smoker groups was classified as having good periodontal health. As shown in Table 3, the significant between-group differences most probably were due to differences in the frequency of the severe stage of PD across the groups, with the lowest frequency of severe PD found among those in the NSC group (15.6\%), followed in increasing order of frequency among those in the NSDM group (28.1\%) and SC group (31.3\%), peaking in the SDM group $(62.5 \%)$. The other stages of PD did not show such notable between-group differences (apart from the already mentioned healthy stage, but the frequency of this stage was quite low in all groups). It is remarkable that while the stages of PD followed a normal distribution in the healthy, non-smoking controls, smoking seemed to cause a shift toward the middle of the severity spectrum, and when smoking was combined with DM, a marked shift toward the most severe stage was observed (Fig. 1).

\section{Diabetes Type, Glycemic Control and Periodontal Status}

There was no significant association between the type of diabetes (T1D or T2D) and periodontal status $\left(\chi^{2}=6.190, p=0.103\right)$, and the distribution of the four stages of periodontal status was almost the same in these two diabetic subgroups, with the exception that severe PD was $17.5 \%$ more frequent in the T2D subgroup than in the T1D subgroup. However, T2D was over-represented in the study sample $\left(n_{\text {type2- }}\right.$ $=42$ vs. $n_{\text {type } 1}=22$ ), so the validity of this observation cannot be established with safety. There was also no significant association between diabetes control and the severity of PD $\left(\chi^{2}=15.503, p=0.078\right)$, but the results of the analysis suggest that the frequency of the severe stage increased with the worsening of diabetes control $\quad\left(n_{\text {well }} \quad\right.$ controlled $=6 ; \quad n_{\text {moderate }}=10$; $n_{\text {mpoorly controlled }}=13$; see Table 4 ).

\section{Number of Teeth}

Pairwise comparisons (Tukey's HSD [honestly significant difference] test) indicated that patients in the SDM group had significantly fewer teeth (mean \pm SD: $16.0 \pm 7.9$ ) than subjects in the NSC group $(20.7 \pm 5.6)$ at $p=0.02$. No other comparison returned a significant result. There was stepwise difference in the mean number of teeth between groups, with patients in the SDM group having the lowest mean number of teeth and subjects in the NSC group having the highest (see also Table 1). In terms of the number of teeth by DM type, patients with T1D had significantly more teeth than patients with T2D $(22 \pm 6.5$ vs. $16.2 \pm 7.7$; $p=0.01)$.

\section{DISCUSSION}

It is well established in the literature that smoking is a major risk factor for developing and aggravating DM and its complications. This is especially true for $\mathrm{T} 2 \mathrm{D}$, the most common form of DM worldwide [62, 63]. It is also known that PD is a frequent sequela of diabetes, so much so that Harald Löe termed it "the sixth complication of diabetes mellitus" [23]. In this study we sougth to answer the question "what effect does smoking have on this specific complication" by analyzing the periodontal status of both smoking and non-smoking patients with DM.

The results are clear in the sense that that while periodontal status followed a normal distribution in healthy, non-smoking controls, smoking caused a shift toward the middle of the PD spectrum, and when smoking was combined with diabetes, a marked shift toward the most severe stage was seen. This result was expected, as it is known that smoking damages the periodontal tissues even in otherwise healthy persons and has a detrimental effect on the progression of PD [64]. We considered it therefore safe to hypothesize that smoking would turn out to be even more detrimental when the primary disease, itself known to have the ability to initiate (and probably aggravate) PD, is present. Within the limits of the present study, we 
believe we have verified this hypothesis. However, the data gathered in this study do not allow an explanation of how exactly DM and smoking interact to aggravate PD. As such, the results may be interpreted as evidence to support the existence of such a detrimental interaction.

We also hypothesized that the periodontal status of diabetic patients with no smoking history would be significantly poorer than that of healthy non-smoking controls. We believed that this hypothesis was logical based on the established relationship between DM and PD. However, it would appear that the results of our study fail to bear this out; for example, there was no remarkable difference between the NSC and NSDM groups (except for the early stage, but not in general). We do not believe that these results should be interpreted as evidence against the well-established relationship; rather, the explanation most likely lies in technical factors and is related to the method of assessment and the different ways in which DM and smoking exert their detrimental effect on the periodontium. Smoking, in fact, can be regarded as repeated episodes of an aggressive chemical attack against the tissues. Smoking damages the periodontal tissues in both systemic and local ways [64], with smoking-induced periodontal inflammation always being the more severe type. Diabetes, on the other hand, develops over a course of time and seems to exert its effects on periodontal tissues effects more slowly and in finer, less direct ways. To give just one expressive example, the antibacterial capacity of the saliva can be downregulated in persons with DM over time, which favors the growth of periodontopathogenic bacterial species [65]. The end result is that while smoking has a rapid effect on the periodontium and maintains a high level of severe inflammation through repeated exposure of these tissues to toxic chemicals, severe stages of periodontal inflammation in DM develop later in the course of the disease and the inflammation is milder than that induced by smoking. As the classification we used measures the actual severity of the periodontal inflammatory process, it is not surprising that it cannot show the fine DM-related difference as efficiently as the massive difference caused by smoking. Still, it should be noted that the classification does not fail to capture the effect of DM entirely: our results clearly show that when smoking is paired up with DM, the destruction of the periodontium is significantly more severe. In summary, DM has a damaging effect on the periodontium, but the clinical staging we used to assess the severity of the inflammation is less sensitive to the effects of DM. While this might be regarded as a weakness of the study, in our professional opinion the classification of Fernandes and colleagues [16] is still the best available classification for study purposes, as it is easy to use and interpret and has a strong positive correspondence to the histopathology of PD.

In terms of the effect of diabetes type, our results suggest that $\mathrm{T} 2 \mathrm{D}$ may be more damaging to the periodontium than T1D, as reflected by the mean number of teeth and the relatively higher prevalence of the most severe stage in participants with T2D. However, the sample size was not large enough for us to allow firm conclusions and, in addition, T2D was over-represented (which is realistic but not optimal for the comparison of the two types). As for the lower number of teeth in patients with T2D, it cannot be excluded that the observed tooth loss is the result of lifestyle rather than the DM itself, given that an unhealthy diet is a major risk factor for the development of T2D [66]. We suggest that this is an interesting finding which may be worth pursuing in future studies, but our results are not sufficient to discuss this topic in any detail.

Glycemic control is another factor which was found not to significantly influence the severity of PD (or vice versa), although the descriptive statistics clearly show that glycemic control became poorer with increasingly severe stage of PD. Taken together, it would appear that there is an effect of glycemic control on PD (in either direction), but that this effect is either statistically non-significant or significant but with too small an effect size to show significance in this sample size. The literature is divided over this question but leans more toward the no-effect side. A number of earlier studies with sample sizes similar to our sample size, 
such as the study of Fernandes and colleagues [16], have also failed to show a significant association between PD severity and glycemic control. In their review, Taylor and colleagues also concluded that there is no such connection [67]. However, there are also studies supporting the counter-argument, providing strong evidence that HbA1c improved as a result of periodontal treatment [43, 68]. A possible explanation for the obvious uncertainty of thes results is that while HbA1c is a well-established clinical parameter that helps treatment, it primarily allows the assessment of the risk of microvascular complications [69]. While microvascular factors may contribute to the deterioration of periodontal status in DM, PD is still predominantly a disease of immunological/ microbiological origin. In short, while our results seem to support the existence of a weak connection, HbA1c might not be the best parameter to link DM with PD. It is safe to assume that results from continuous glucose monitoring would allow a much more decisive conclusion, but we did not have that option. This is definitely a limitation to our study, but in Hungary, continuous monitoring is not widespread and, consequently, collecting data on even the relatively small number of patients with regularly recorded continuous monitoring data was extremely difficult. In contrast, HbA1c is routinely and meticulously recorded for all patients receiving diabetes care. We had to choose between reporting the routinely recorded standard parameter or omitting glycemic control altogether, and the latter did not appear to be the adequate choice in the given context.

As often happens, our study has resulted in more questions than it can answer, but we believe that we can draw at least one valid conclusion: smoking damages even the healthy periodontium, but when a DM patient smokes, the destruction multiplies. This is quite similar to what our research group described about the relationship between smoking and PD in psoriasis [11]. In that study, we concluded that smoking and psoriasis act in synergy to boost periodontal inflammation, which in turn leads to the over-representation of the most severe stage in smoking patients. The situation appears to be the same in the case of diabetes, i.e. the relatively mild damaging effect of the disease itself is multiplied by the repeated chemical challenge of cigarette smoke exposure. How this effect is realized goes beyond the scope of this study, but it is safe to assume that the process has both microvascular and immunological components, and it is probably the meeting of these different pathogenic pathways that gives rise to the observed massive deterioration. Some studies have addressed the issue of the effect of smoking and DM on periodontal health [50-52], but none of these concentrated explicitly on the interaction of the two factors. Our results are new in this respect and demonstrate another way in which smoking can do further damage to the health of patients with DM.

\section{CONCLUSIONS}

Within the limitations of this study, we conclude that smoking damages even the healthy periodontium, but when a DM patient smokes, the destruction caused by smoking is multiplied, while the effect of DM alone is relatively mild. These results might be interpreted as indicative of a synergy between DM and smoking to damage the periodontal tissues, but standing alone they do not allow us to draw a hard conclusion, especially as the size of the study sample did not allow more in-depth analyses. The details thus remain to be clarified. Nevertheless, based on these results, we conclude that the increased risk of severe PD is another reason why patients with DM should be discouraged from smoking.

\section{ACKNOWLEDGEMENTS}

The authors would like to express their gratitude to Dr. Gábor Braunitzer for his critical comments on the manuscript. We would like to thank the participants of the study for their time and cooperation. 
Funding. No funding was received for this research. The Rapid Service Fee was funded by the OA funding of the University of Szeged.

Authorship. All named authors meet the International Committee of Medical Journal Editors (ICMJE) criteria for authorship for this article, take responsibility for the integrity of the work as a whole, and have given their approval for this version to be published.

Authorship Contributions. MA designed the research and coordinated the research team; EB, DG and $\mathrm{SN}$ performed patient evaluation and collected patient data; CL, GE, ZB and TV analyzed the data and drafted the manuscript; EB, DG and MA wrote and made the final version of the manuscript. All authors have read and approved the final manuscript.

Disclosures. Emese Battancs, Dorottya Gheorghita, Szabolcs Nyiraty, Csaba Lengyel, Gabriella Eördegh, Zoltán Baráth, Tamás Várkonyi and Márk Antal have nothing to disclose.

Compliance with Ethics Guidelines. Participation was voluntary, and written informed consent was obtained from each participant. The protocol and design of the study conformed in all respects to the tenets of the Declaration of Helsinki of 1964, and its later amendments, and the study was approved by the Regional Research Ethics Committee for Medical Research at the University of Szeged, Hungary (144/2014-B/001, accepted 2014, modified in 2019).

Data Availability. The datasets generated during and/or analyzed during the current study are available from the corresponding author on reasonable request.

Open Access. This article is licensed under a Creative Commons Attribution-NonCommercial 4.0 International License, which permits any non-commercial use, sharing, adaptation, distribution and reproduction in any medium or format, as long as you give appropriate credit to the original author(s) and the source, provide a link to the Creative Commons licence, and indicate if changes were made. The images or other third party material in this article are included in the article's Creative Commons licence, unless indicated otherwise in a credit line to the material. If material is not included in the article's Creative Commons licence and your intended use is not permitted by statutory regulation or exceeds the permitted use, you will need to obtain permission directly from the copyright holder. To view a copy of this licence, visit http://creativecommons.org/licenses/by$\mathrm{nc} / 4.0 /$.

\section{REFERENCES}

1. Molloy J, Wolff LF, Lopez-Guzman A, Hodges JS. The association of periodontal disease parameters with systemic medical conditions and tobacco use. J Clin Periodontol. 2004;31(8):625-32.

2. Aarabi G, Schnabel RB, Heydecke G, Seedorf U. Potential impact of oral inflammations on cardiac functions and atrial fibrillation. Biomolecules. 2018;8(3):66. biom8030066. https://doi.org/10.3390/

3. Cheng Z, Meade J, Mankia K, Emery P, Devine DA. Periodontal disease and periodontal bacteria as triggers for rheumatoid arthritis. Best Pract Res Clin Rheumatol. 2017;31(1):19-30.

4. Heredia PA, Lafaurie GI, Bautista-Molano W, et al. Predictive factors related to the progression of periodontal disease in patients with early rheumatoid arthritis: a cohort study. BMC Oral Health. 2019;19(1):240.

5. Loutan L, Alpizar-Rodriguez D, Courvoisier DS, Finckh A, Mombelli A, Giannopoulou C. Periodontal status correlates with anti-citrullinated protein antibodies in first-degree relatives of individuals with rheumatoid arthritis. J Clin Periodontol. 2019;46(7):690-8.

6. Ryan ME, Carnu O, Kamer A. The influence of diabetes on the periodontal tissues. J Am Dent Assoc. 2003;134 Spec No:34S-40S. https://doi.org/10. 14219/jada.archive.2003.0370.

7. Linden GJ, Lyons A, Scannapieco FA. Periodontal systemic associations: review of the evidence. J Clin Periodontol. 2013;40(Suppl 14):S8-19.

8. Tsioufis C, Kasiakogias A, Thomopoulos C, Stefanadis C. Periodontitis and blood pressure: the 
concept of dental hypertension. Atherosclerosis. 2011;219(1):1-9.

9. Mashalkar VN, Suragimath G, Zope SA, Varma SA. A cross-sectional study to assess and correlate osteoporosis and periodontitis among postmenopausal women: a dual energy X-ray absorptiometry study. J Midlife Health. 2018;9(1):2-7.

10. Yamada J, Amar S, Petrungaro P. Psoriasis-associated periodontitis: a case report. J Periodontol. 1992;63(10):854-7.

11. Antal M, Braunitzer G, Mattheos N, Gyulai R, Nagy K. Smoking as a permissive factor of periodontal disease in psoriasis. PLoS ONE. 2014;9(3):e92333.

12. Hobbins S, Chapple IL, Sapey E, Stockley RA. Is periodontitis a comorbidity of COPD or can associations be explained by shared risk factors/behaviors? Int J Chron Obstruct Pulmon Dis. 2017;12: 1339-49.

13. Radnai M, Gorzo I, Nagy E, Urban E, Novak T, Pal A. A possible association between preterm birth and early periodontitis. A pilot study. J Clin Periodontol. 2004;31(9):736-41.

14. Marakoglu I, Gursoy UK, Marakoglu K, Cakmak H, Ataoglu T. Periodontitis as a risk factor for preterm low birth weight. Yonsei Med J. 2008;49(2):200-3.

15. Battancs E, Gorzo I, Pal A, et al. Pregnant women's oral hygiene knowledge and habits after the second millennium in South-East Hungary. Fogorv Sz. 2011;104(3):75-9.

16. Fernandes JK, Wiegand RE, Salinas CF, et al. Periodontal disease status in gullah african americans with type 2 diabetes living in South Carolina. J Periodontol. 2009;80(7):1062-8.

17. Seymour GJ, Powell RN, Davies WI. The immunopathogenesis of progressive chronic inflammatory periodontal disease. J Oral Pathol. 1979;8(5):249-65.

18. Hirschfeld J, Howait M, Movila A, et al. Assessment of the involvement of the macrophage migration inhibitory factor-glucocorticoid regulatory dyad in the expression of matrix metalloproteinase- 2 during periodontitis. Eur J Oral Sci. 2017;125(5): 345-54.

19. Kalakonda B, Koppolu P, Baroudi K, Mishra A. Periodontal systemic connections-novel associations-a review of the evidence with implications for medical practitioners. Int J Health Sci (Qassim). 2016;10(2):293-307.
20. Graves DT, Kayal RA. Diabetic complications and dysregulated innate immunity. Front Biosci. 2008;13:1227-399.

21. Pham MN, Hawa MI, Pfleger C, et al. Pro- and antiinflammatory cytokines in latent autoimmune diabetes in adults, type 1 and type 2 diabetes patients: action LADA 4. Diabetologia. 2011;54(7): 1630-8.

22. The Emerging Risk Factors Collaboration, Sarwar N, Gao P, et al. Diabetes mellitus, fasting blood glucose concentration, and risk of vascular disease: a collaborative meta-analysis of 102 prospective studies. Lancet. 2010;375(9733):2215-22.

23. Loe H. Periodontal disease. The sixth complication of diabetes mellitus. Diabetes Care. 1993;16(1): 329-34.

24. Graziani F, Gennai S, Solini A, Petrini M. A systematic review and meta-analysis of epidemiologic observational evidence on the effect of periodontitis on diabetes an update of the EFP-AAP review. J Clin Periodontol. 2018;45(2):167-87.

25. Baeza M, Morales A, Cisterna C, et al. Effect of periodontal treatment in patients with periodontitis and diabetes: systematic review and meta-analysis. J Appl Oral Sci. 2020;28:e20190248.

26. Mataftsi M, Koukos G, Sakellari D. Prevalence of undiagnosed diabetes and pre-diabetes in chronic periodontitis patients assessed by an HbA1c chairside screening protocol. Clin Oral Investig. 2019;23(12):4365-70.

27. Lappin DF, Eapen B, Robertson D, Young J, Hodge PJ. Markers of bone destruction and formation and periodontitis in type 1 diabetes mellitus. J Clin Periodontol. 2009;36(8):634-41.

28. Salvi GE, Franco LM, Braun TM, et al. Pro-inflammatory biomarkers during experimental gingivitis in patients with type 1 diabetes mellitus: a proof-ofconcept study. J Clin Periodontol. 2010;37(1):9-16.

29. Sanz M, Ceriello A, Buysschaert M, et al. Scientific evidence on the links between periodontal diseases and diabetes: Consensus report and guidelines of the joint workshop on periodontal diseases and diabetes by the International Diabetes Federation and the European Federation of Periodontology. J Clin Periodontol. 2018;45(2):138-49.

30. Lim LP, Tay FB, Sum CF, Thai AC. Relationship between markers of metabolic control and inflammation on severity of periodontal disease in patients with diabetes mellitus. J Clin Periodontol. 2007;34(2):118-23. 
31. Taboza ZA, Costa KL, Silveira VR, et al. Periodontitis, edentulism and glycemic control in patients with type 2 diabetes: a cross-sectional study. BMJ Open Diabetes Res Care. 2018;6(1):e000453.

32. Lalla E, Cheng B, Lal S, et al. Diabetes mellitus promotes periodontal destruction in children. J Clin Periodontol. 2007;34(4):294-8.

33. Hodge PJ, Robertson D, Paterson K, Smith GL, Creanor S, Sherriff A. Periodontitis in non-smoking type 1 diabetic adults: a cross-sectional study. J Clin Periodontol. 2012;39(1):20-9.

34. Costa FO, Miranda Cota LO, Pereira Lages EJ, et al. Progression of periodontitis and tooth loss associated with glycemic control in individuals undergoing periodontal maintenance therapy: a 5-year follow-up study. J Periodontol. 2013;84(5):595-605.

35. Garcia D, Tarima S, Okunseri C. Periodontitis and glycemic control in diabetes: NHANES 2009 to 2012. J Periodontol. 2015;86(4):499-506.

36. Kim EK, Lee SG, Choi YH, et al. Association between diabetes-related factors and clinical periodontal parameters in type- 2 diabetes mellitus. BMC Oral Health. 2013;13:64.

37. Preshaw PM, Alba AL, Herrera D, et al. Periodontitis and diabetes: a two-way relationship. Diabetologia. 2012;55(1):21-31.

38. Casanova L, Hughes FJ, Preshaw PM. Diabetes and periodontal disease: a two-way relationship. $\mathrm{Br}$ Dent J. 2014;217(8):433-7.

39. Taylor GW. Bidirectional interrelationships between diabetes and periodontal diseases: an epidemiologic perspective. Ann Periodontol. 2001;6(1):99-112.

40. Correa FO, Goncalves D, Figueredo CM, Bastos AS, Gustafsson A, Orrico SR. Effect of periodontal treatment on metabolic control, systemic inflammation and cytokines in patients with type 2 diabetes. J Clin Periodontol. 2010;37(1):53-8.

41. Engebretson S, Kocher T. Evidence that periodontal treatment improves diabetes outcomes: a systematic review and meta-analysis. J Clin Periodontol. 2013;40(Suppl 14):S153-S163163.

42. Koromantzos PA, Makrilakis K, Dereka X, Katsilambros N, Vrotsos IA, Madianos PN. A randomized, controlled trial on the effect of non-surgical periodontal therapy in patients with type 2 diabetes. Part I: effect on periodontal status and glycaemic control. J Clin Periodontol. 2011;38(2):142-7.

43. Quintero AJ, Chaparro A, Quirynen M, et al. Effect of two periodontal treatment modalities in patients with uncontrolled type 2 diabetes mellitus: a randomized clinical trial. J Clin Periodontol. 2018;45(9):1098-106.

44. Kocher T, Holtfreter B, Petersmann A, et al. Effect of periodontal treatment on HbA1c among patients with prediabetes. J Dent Res. 2019;98(2):171-9.

45. Parker DR, Fallone D, Martin RA, et al. The relation between smoking status and medical conditions among incarcerated adults. J Addict Med. 2014;8(2): $90-5$.

46. Heydarpour P, Manouchehrinia A, Beiki O, et al. Smoking and worsening disability in multiple sclerosis: a meta-analysis. Acta Neurol Scand. 2018;138(1):62-9.

47. Fagard RH, Nilsson PM. Smoking and diabetes-the double health hazard! Prim Care Diabetes. 2009;3(4):205-9.

48. Cignarelli M, Lamacchia O, Di Paolo S, Gesualdo L. Cigarette smoking and kidney dysfunction in diabetes mellitus. J Nephrol. 2008;21(2):180-9.

49. Obradovic R, Kesic LJ, Gasic J, Petrovic M, Zivkovic $\mathrm{N}$. Role of smoking in periodontal disease among diabetic patients. West Indian Med J. 2012;61(1): 98-101.

50. Gupta N, Gupta ND, Garg S, et al. The effect of type 2 diabetes mellitus and smoking on periodontal parameters and salivary matrix metalloproteinase- 8 levels. J Oral Sci. 2016;58(1):1-6.

51. Orbak R, Tezel A, Canakci V, Demir T. The influence of smoking and non-insulin-dependent diabetes mellitus on periodontal disease. J Int Med Res. 2002;30(2):116-25.

52. Javed F, Al-Kheraif AA, Salazar-Lazo K, et al. Periodontal inflammatory conditions among smokers and never-smokers with and without type 2 diabetes mellitus. J Periodontol. 2015;86(7):839-46.

53. Antal M, Battancs E, Bocskai M, Braunitzer G, Kovacs L. An observation on the severity of periodontal disease in past cigarette smokers suffering from rheumatoid arthritis- evidence for a long-term effect of cigarette smoke exposure? BMC Oral Health. 2018;18(1):82.

54. Genco RJ, Borgnakke WS. Risk factors for periodontal disease. Periodontol 2000. 2013;62(1): 59-94.

55. Greene JC, Vermillion JR. The simplified oral hygiene index. J Am Dent Assoc. 1964;68:7-13.

56. Faul F, Erdfelder E, Lang AG, Buchner A. G*Power 3: a flexible statistical power analysis program for the 
social, behavioral, and biomedical sciences. Behav Res Methods. 2007;39(2):175-91.

57. American DA. Diagnosis and classification of diabetes mellitus. Diabetes Care. 2011;34(Suppl 1): S62-S69.

58. Lin CY, Chen F, Hariri A, et al. Photoacoustic imaging for noninvasive periodontal probing depth measurements. J Dent Res. 2018;97(1):23-30.

59. Leroy R, Eaton KA, Savage A. Methodological issues in epidemiological studies of periodontitis-how can it be improved? BMC Oral Health. 2010;10:8.

60. Souto MLS, Rovai ES, Ganhito JA, Holzhausen M, Chambrone L, Pannuti CM. Efficacy of systemic antibiotics in nonsurgical periodontal therapy for diabetic subjects: a systematic review and metaanalysis. Int Dent J. 2018;68(4):207-20.

61. Nibali L, Krajewski A, Donos N, et al. The effect of furcation involvement on tooth loss in a population without regular periodontal therapy. J Clin Periodontol. 2017;44(8):813-21.

62. Chang SA. Smoking and type 2 diabetes mellitus. Diabetes Metab J. 2012;36(6):399-403.

63. Haire-Joshu D, Glasgow RE, Tibbs TL. Smoking and diabetes. Diabetes Care. 1999;22(11):1887-988.
64. Leite FRM, Nascimento GG, Scheutz F, Lopez R. Effect of smoking on periodontitis: a systematic review and meta-regression. Am J Prev Med. 2018;54(6):831-41.

65. Huang J, Xiao Y, Xu A, Zhou Z. Neutrophils in type 1 diabetes. J Diabetes Investig. 2016;7(5):652-63.

66. Abdullah A, Peeters A, de Courten M, Stoelwinder J. The magnitude of association between overweight and obesity and the risk of diabetes: a meta-analysis of prospective cohort studies. Diabetes Res Clin Pract. 2010;89(3):309-19.

67. Taylor JJ, Preshaw PM, Lalla E. A review of the evidence for pathogenic mechanisms that may link periodontitis and diabetes. J Clin Periodontol. 2013;40(Suppl 14):S113-S134.

68. Mauri-Obradors E, Merlos A, Estrugo-Devesa A, Jane-Salas E, Lopez-Lopez J, Vinas M. Benefits of non-surgical periodontal treatment in patients with type 2 diabetes mellitus and chronic periodontitis: a randomized controlled trial. J Clin Periodontol. 2018;45(3):345-53.

69. World Health Organization. Use of glycated haemoglobin (HbA1c) in the diagnosis of diabetes mellitus. Abbreviated report of a WHO consultation. Geneva: World Health Organization; 2011. 